AperTO - Archivio Istituzionale Open Access dell'Università di Torino

Wastewater bioremediation using white rot fungi: validation of a dynamical system with real data obtained in laboratory

This is a pre print version of the following article:

Original Citation:

Availability:

This version is available http://hdl.handle.net/2318/1661185

since 2018-12-13T15:53:49Z

Published version:

DOI:10.1002/mma.4834

Terms of use:

Open Access

Anyone can freely access the full text of works made available as "Open Access". Works made available under a Creative Commons license can be used according to the terms and conditions of said license. Use of all other works requires consent of the right holder (author or publisher) if not exempted from copyright protection by the applicable law. 


\title{
Wastewater bioremediation using white rot fungi: validation of a dynamical system with real data obtained in laboratory
}

\author{
Iulia Martina Bulai ${ }^{a}$, Federica Spina ${ }^{b}$, Giovanna Cristina Varese $^{b}$, Ezio Venturino \\ Dipartimento di Matematica "Giuseppe Peano", Università di Torino, \\ via Carlo Alberto 10, 10123 Torino, Italia; \\ Member of the INdAM research group GNCS \\ ${ }^{a}$ Department of Information Engineering, \\ University of Padova. Via Gradenigo, 6/B, \\ 35131, Padova, Italy; Member of the INdAM research group GNCS \\ ${ }^{b}$ Dipartimento di Scienze della Vita e Biologia dei Sistemi, Università di Torino, \\ viale Mattioli 25, 10125 Torino, Italia.
}

December 13, 2018

\begin{abstract}
Nowadays wastewater treatment has become an important issue in view of the ever increasing worldwide paucity of abundant and clean water supplies. In this work, we propose a mathematical model describing the process of decolourisation of textile industry wastewater and validate it using data from a laboratory experiment. To this aim a selected white rot fungus, capable of degrading a wide range of recalcitrant compounds, is used against Remazol Brilliant Blue Reactive dye (RBBR). The real data obtained in laboratory are used to fit the parameters of our model. The qualitative analysis is performed to study the behaviour of the wastewater and of the fungus as functions of time. In the present study the carbon (glucose) has an important role in the system since it can sustain the fungal metabolism and growth. Furthermore a more general mathematical model is studied, considering an open system where there is a constant input and output of pollutant and nutrients respectively.

Keywords: dynamical system; biodegradation; wastewater; fungi; parameter fitting
\end{abstract}

\section{Introduction}

In the EU market, textile industry represents a traditional industrial sector. In 2013 this sector had a turnover of 166 billion euro, more than 185, 000 working factories, and 1.7 million people employed. Five countries (Italy, Germany, UK, France and Spain) cover most of the European market, of which about $20 \%$ 
is sold outside the EU border. In recent years, this industrial sector has been subjected to radical transformations, having to face emerging countries and the increasing environmental and economic issues. One of the main problems of textile industries is the high water consumption, even $30-50 \mathrm{l}$ for each $\mathrm{kg}$ of cloth, [1]. Since clean water is worldwide recognized as a limiting resource, the reduction of water use and its perfect re-cycling in the manufacturing chain will enhance the sustainability of the textile process. Its competitiveness will also be enhanced by hindering the massive water pollution due to the discharge of colored wastewaters into the hydric basin.

When dyes or their metabolites enter into the water cycle, they may accumulate and finally affect the aquatic organisms as well as human society. The need of effective treatments is strengthened by the unfortunate fact that classical technologies cannot solve the environmental issue [1]. Being economic and environmental sustainable, biological treatments are receiving more and more attention from scientific community. Fungi are well known not only as decomposers of lignin but also as bioremediation agents of industrial wastewaters, [2]. Many reports have demonstrated that they are capable of transforming dyes in colorless and harmless substances, [3]. A number of strains mostly belonging to Basidiomycetes have been successfully used against dyes as well as textile wastewaters, [4], but there are still some limitations for their application at large scale. Although fungi are known to be versatile and robust organisms, they may be affected by the extreme environmental conditions of operative wastewaters treatment plants. The toxicity of wastewaters and the absence of source of nourishment are among the most limiting factors. For instance fungi can be affected by the concentration of the dye itself, ultimately reducing their growth and activity, [5]. Nutrients instead play a double role on fungal metabolism: i) the carbon source is needed for mycelium development allowing to hypothesize a growing biomass and a long-lasting process; ii) it plays a crucial role in the activation of secondary metabolism at the base of the production of oxidative enzymes, causing a significant enhancement of decolorization yields, [6]. Despite the several studies made throughout the past years, the relation between carbon and fungal performance has been described mostly by empirical studies. This work brings new knowledge about the role of carbon source in the bioremediation processes catalyzed by fungi. In this paper we adopt a different focus from what has been previous done in the literature, $[8,9]$, namely we consider the importance of the nutrients in the biodegradation process of organic pollutants, e.g. Remazol Brilliant Blue Reactive dye by Trametes pubescens, [7], and not anymore the role of the dissolved oxygen that can be relevant in the biodegradation process using algae [10].

In the next Section, we first present the model for a closed system, in Section 3 we present its qualitative analysis. Section 4 contains the model validation, while in Section 5 and 6 respectively a more general model is introduced, for an open system, where a constant input and output of pollutant and carbon is considered, and its qualitative analysis is performed. A final discussion concludes the paper. 


\section{The mathematical model used to fit the data}

In this section we introduce the mathematical model that will be used to fit the real data obtained in the laboratory experiment. It is composed by three differential equations respectively representing the organic pollutant concentration, $P$, the biomass of the Trametes pubescens fungus, $F$, and the source of nutrients (of which we measure the glucose), $C$. At this point of the analysis we work with a closed system in which no inputs or outputs are included. Later, after the validation of the model, a more general system will be studied, accounting for a constant input of both pollutants and nutrients. The model is:

$\frac{d P}{d t}=-a_{1} \frac{P}{h_{P}+P} F-a_{P} \frac{C}{h_{C}+C} \frac{P}{h_{P}+P} F$

$\frac{d F}{d t}=e a_{1} \frac{P}{h_{P}+P} F+e a_{P} \frac{C}{h_{C}+C} \frac{P}{h_{P}+P} F+f k_{C} \frac{C}{h_{C}+C} F$

$\frac{d C}{d t}=-k_{C} \frac{C}{h_{C}+C} F+\left(a_{C}-1\right) a_{1} \frac{P}{h_{P}+P} F+\left(a_{C}-1\right) a_{P} \frac{C}{h_{C}+C} \frac{P}{h_{P}+P} F$.

In the first equation of the system (1) the behaviour in time of the pollutant $P$ is described. The pollutant is degraded into colourless metabolite by the fungus. Parts of this compounds are potentially beneficial for the fungus and are therefore assimilated. In this way, then, a fraction of the pollutant may contribute to the growth of the fungus. Trametes pubescens feeds on it. We distinguish between the two cases, where this process does not need glucose, the first term of the RHS of the first equation, or needs it, the second term respectively. The rate of biodegradation is $a_{1}+a_{P} C$. The specific growth rate of $F$, corresponding to pollutants' biodegradation, depends on $P$ or on both $P$ and $C$, respectively. To model the fact that glucose is used directly by $F$ in the biodegradation process, we use the product of Monod equations both for $C$ and for $P,[13,14]$; in other words, we express the fungal growth rate in terms of substrate concentrations. A "Double Monod" model proposed in [16] can be used when two substrates are involved. It is known that under proper conditions the presence of glucose increases the efficiency of decolourization process [19]. Otherwise where the glucose is not directly used in the biodegradation process only a Monod equation is used for $P$. In fact some microorganisms, such as bacteria and fungi, can grow by using pollutants as their carbon and/or energy source, [15].

The second equation models the fungi growth due to the above mentioned mechanism, i.e. their interaction with $P$, or with both $P$ and $C$. In the first term, $e$ denotes a conversion factor, $e \in[0,1]$. Fungi, as living organisms, need nutrients to survive, and the $C$ source that we added in the present study was aimed at sustaining the growth and the metabolism of the fungus. It is well known that modifications of the cultural medium can affect the rate and the yield of the biomass development, [17]. For the fungi population it is "easier" to use nutrients, that are ready to be assimilated, than the pollutants, which need to be decomposed before they can be used. We assume that fungi can also grow by assimilating only nutrients, a process that occurs at rate $k_{C}$, expressed 
by the third term in the equation. Here we find a second conversion factor, $f \in[0,1]$. Note that we do not include any fungal mortality here, because the duration of the experiment is just 10 days. In view of this very short time span, no functional inactivity or mortality of the fungal population is needed in the model.

Finally in the last equation the nutrients are used by the fungus, the uptake occuring at rate $k_{C}$. The second and third terms express the fact that for the degradation of $P$ the nutrients are not used at all, second term, or are instead employed by the fungus to perform this process, third term, in which case they are consumed at rate $a_{P} C$. On the other hand, in the degradation process, the fungal oxidative cascade converts $P$ into other molecules that could be used by $F$ for its metabolism. We assume that this second process occurs at rate $a_{C}$, thereby increasing the $C$ concentration. All the parameters of the model are non-negative.

In summary, the system behaves so that interaction among $P, F$ and $C$ leads to the decrease of $P$ by the active metabolism of $F$ consuming the available $C$.

\section{Analysis}

We summarize here the theoretical results on the equilibria of the system (1).

\section{Proposition 1.}

1. The trivial equilibrium point, $E_{0}=(0,0,0)$, exists always. The eigenvalues of the Jacobian matrix evaluated at $E_{0}$ are $\lambda_{i}=0$, for $i=1,2,3$.

2. The fungi-and-glucose-free point, $E_{1}=(P, 0,0)$, exists always. Two of the eigenvalues of the Jacobian at this point are $\lambda_{i}=0$, for $i=1,2$, the third one is

$$
\lambda_{3}=e a_{1} \frac{P}{h_{P}+P}>0 .
$$

Hence $E_{1}$ is unconditionally unstable.

3. The pollutant-and-glucose-free point, $E_{2}=(0, F, 0)$, exists always. One of the eigenvalue of the Jacobian at this point is $\lambda_{1}=0$, while

$$
\lambda_{2}=-a_{1} \frac{F}{h_{P}} \quad \text { and } \quad \lambda_{3}=-k_{C} \frac{F}{h_{C}} .
$$

Thus these eigenvalues do not require any condition for stability.

4. The pollutant-fungi-free point, $E_{3}=(0,0, C)$, exists always. Again two eigenvalues are zero $\lambda_{i}=0$, for $i=1,2$, the third one is

$$
\lambda_{3}=f k_{C} \frac{C}{h_{C}+C}>0 .
$$

Hence $E_{3}$ is unconditionally unstable. 
5. The fungi-free point, $E_{4}=(P, 0, C)$, exists always. We have once more $\lambda_{i}=0$, for $i=1,2$, and

$$
\lambda_{3}=e a_{1} \frac{P}{h_{P}+P}+e a_{P} \frac{C}{h_{C}+C} \frac{P}{h_{P}+P}+f k_{C} \frac{C}{h_{C}+C}>0 .
$$

Thus $E_{4}$ is always unstable.

Proof: The existence of the equilibria $E_{i}, i=0,1, \ldots, 4$ is trivial. For the stability we compute the Jacobian matrix associated to system (1):

$$
J=\left[\begin{array}{ccc}
J_{11} & J_{12} & J_{13} \\
-e J_{11} & -e J_{12}+\frac{f k_{C} C}{h_{C}+C} & -e J_{13}+\frac{f k_{C} h_{C} F}{\left(h_{C}+C\right)^{2}} \\
-\left(a_{C}-1\right) J_{11} & -\frac{k_{C} C}{h_{C}+C}-\left(a_{C}-1\right) J_{12} & -\frac{k_{C} h_{C} F}{\left(h_{C}+C\right)^{2}}-\left(a_{C}-1\right) J_{13}
\end{array}\right]
$$

where

$J_{11}=-\frac{a_{1} h_{P} F}{\left(h_{P}+P\right)^{2}}-\frac{a_{P} h_{P} C F}{\left(h_{C}+C\right)\left(h_{P}+P\right)^{2}}, \quad J_{12}=-\frac{a_{1} P}{h_{P}+P}-\frac{a_{P} C P}{\left(h_{C}+C\right)\left(h_{P}+P\right)}, \quad J_{13}=\frac{a_{P} h_{C} P F}{\left(h_{C}+C\right)^{2}\left(h_{P}+\right.}$

and with easy computations we obtain the stated claims.

\section{Model validation}

\subsection{A brief introduction to the biological experiment and data collection}

Trametes pubescens MUT 2400 was previously selected for its dye degrading efficacy associated to a high enzymes production, [12]. It is preserved at the Mycotheca Universitatis Taurinensis Collection (MUT, University of Torino, Department of Life Sciences and Systems Biology) on rich medium (MEA: 20 $\mathrm{g} / \mathrm{l}$ glucose, $20 \mathrm{~g} / \mathrm{l}$ malt extract, $2 \mathrm{~g} / \mathrm{l}$ peptone, $18 \mathrm{~g} / \mathrm{l}$ agar). The liquid cultures were carried out in $100 \mathrm{ml}$ Erlenmeyer flasks, incubated at $24 \mathrm{C}$ and $110 \mathrm{rpm}$ in the dark in an orbital shaker. The culture medium (40 $\mathrm{ml}$ final volume) was made as follow: glucose $C(2 \mathrm{~g} / \mathrm{l})$, peptone as source of nitrogen $(0.2 \mathrm{~g} / \mathrm{l})$ and $P$. The tested organic pollutant $P$ is the anthraquinone dye Remazol Brilliant Blue R (RBBR, Sigma Aldrich), inoculated at 200 ppm (maximal absorbance at $596 \mathrm{~nm})$.

In order to evaluate the role of $C$ on fungal performance, the fungus was inoculated in the presence of only $F$ (line A) in the presence of only $C$ (line B) and in presence of both $P$ and $C$ (line $\mathrm{C}$ ), respectively. The need of an active co-metabolism for pollutants degradation is a well-know phenomenon [18], but it is also true that some fungi may show decolourisation skills even in the absence of any additional $C$ source [19].

In each flask the fungus was inoculated with $4 \mathrm{ml}$ fungal suspension, prepared by homogenizing agar squares derived from the margins of an overgrown colony together with sterile water $\left(1 \mathrm{~cm}^{2} / \mathrm{ml}\right)$ using an Ultraturrax homogenizer (IKA, 
Staufen, Germany). During the 7-day fermentation, at every collection point 3 flasks were sacrificed to carry out the analysis. The absorbance of $P$ was spectrophotometrically measured in the visible range (TECAN Infinite $M 200$, Austria). The $C$ concentration was determined using the 2,4-dinitrosalicyclic acid assay for reducing sugars according to the method of Miller [11]. The fungal biomass $F$ was filtered from the medium and dried at $60 \mathrm{C}$ for $24 \mathrm{~h}$ in order to measure the dry weight.

\subsection{Model validation}

For the numerical simulations we used Matlab R2016a, and we used the TrustRegion-Reflective Least Squares Algorithm to fit the data. This algorithm allows us to put constraints on the parameters, i.e. we can impose the positivity for all of them and furthermore require $e, f \in[0,1]$.

Three cultural lines were set in the laboratory experiments: they contain comparable $F(1-3 \mathrm{mg} / \mathrm{l})$ and $P(0.2 \mathrm{~g} / \mathrm{l})$ but differed for the nutrient content, namely glucose was absent in line A, pollutant was absent in line B while both pollutant and glucose were added in line $\mathrm{C}$.

Line A was set in order to investigate the possible toxicity of $\mathrm{P}$ and the fungal capability to use it as source of nutrient. Organopollutants including dyes can indeed be toxic to microorganisms; this usually happens at higher dyes concentration and eventually using sensitive fungi. In the present study, we were quite confident about the robustness of the fungus due to previous studies and its source of isolation, but it was necessary to validate this assumptions from an objective point of view. As regards line A, the initial conditions (i.c.) of $P$ and $F$ were $0.226 \mathrm{~g} / 1,0.001 \mathrm{~g}$, respectively. The results are summarized in Table 1.

Table 1: The data set for line $A$

\begin{tabular}{c|c|c|c|c|c|c|c|c|c} 
Time $(\mathrm{h})$ & $t_{0}$ & 1.5 & 16.5 & 19.5 & 22.5 & 27 & 41 & 45 & 48 \\
\hline$P(\mathrm{~g} / \mathrm{l})$ & 0.200 & 0.179 & 0.169 & 0.158 & 0.156 & 0.147 & 0.146 & 0.124 & 0.150 \\
$F(\mathrm{~g})$ & $1 * 10^{-3}$ & $1 * 10^{-3}$ & $1 * 10^{-3}$ & $1 * 10^{-3}$ & $1 * 10^{-3}$ & $1 * 10^{-3}$ & $1 * 10^{-3}$ & $3 * 10^{-3}$ & $3 * 10^{-3}$
\end{tabular}

\begin{tabular}{c|c|c|c|c|c|c|c|c} 
Time(h) & 51 & 65 & 69 & 72 & 98.5 & 116 & 188 & 260 \\
\hline$P(\mathrm{~g} / \mathrm{l})$ & 0.119 & 0.065 & 0.051 & 0.056 & 0.044 & 0.033 & 0.017 & 0.016 \\
$F(\mathrm{~g})$ & $7 * 10^{-3}$ & $4 * 10^{-3}$ & $4 * 10^{-3}$ & $5 * 10^{-3}$ & $8 * 10^{-3}$ & $10 * 10^{-3}$ & $4 * 10^{-3}$ & $6 * 10^{-3}$
\end{tabular}

Note that a small amount of $C(0.2 \mathrm{~g} / \mathrm{l})$ was detected at the beginning of line A experiment, and it could be ascribable to residues contained in the inoculum itself. In any case already during the first hours of the experiments it was completely consumed, and any residual $C$ source could not be detected. The findings indicate that the pollutant is not toxic for the fungus, that thrives, although its growth is essentially negligible and slow, about $6-10 \mathrm{mg} / \mathrm{l}$ in one 
week. The pollutant at the end of the experiment is degraded.

Line B was performed in order to evaluate the growth of the fungus due only to glucose source. Line $\mathrm{C}$ was aimed at reaching high and fast degradation of $P$ by $F$, by sustaining the primary metabolism and consequently the secondary one that includes the actual agents of $P$ transformation, the fungal oxidative enzymes. To this end, glucose as $C$ source $(2 \mathrm{~g} / \mathrm{l})$ was added in this line $\mathrm{C}$ in the same quantity as in line B; the concentration was minimal but able to guarantee the proper functioning of the minimal primary metabolism of the fungus. The pollutant is finally degraded, its values being comparable to those of line A at the end of the week. But the difference lies in the much faster reduction rate, due here to the presence of $C$. It is enough to look at the concentration value after one day, to see that it is halved, while in line $\mathrm{A}$ it still remains quite high.

We use the data of the three different lines in order to fit all the parameters of the closed system (1). The model in the absence of $C$ simplifies to

$$
\frac{d P}{d t}=-a_{1} \frac{P F}{h_{P}+P}, \quad \frac{d F}{d t}=e a_{1} \frac{P F}{h_{P}+P},
$$

which has the equilibrium $(0, F(\infty))$ and furthermore $e P+F=e P(0)+F(0)=$ $e P(\infty)+F(\infty)=0+F(\infty)$, so that $F(\infty)$ is known. Thus the conversion factor is

$$
e=\frac{F(\infty)-F(0)}{P(0)}=0.022 .
$$

The fitting of the dataset from line A allows then the determination of the parameters

$$
a_{1}=664(l s)^{-1}, \quad h_{P}=100 \mathrm{~g} / \mathrm{l} .
$$

In Figure $1 P$ and $F$ are shown respectively for $260 \mathrm{~h}$ (10 days), with the red stars representing the real data collected in laboratory, while the blue continuous lines indicate the optimal solutions of the system, (4).
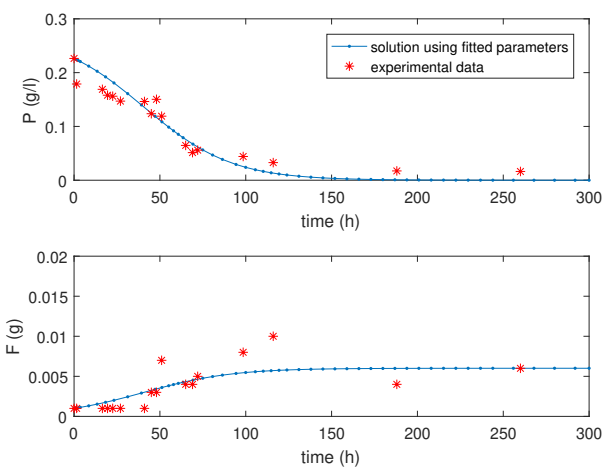

Figure 1: The solution of (1) using the parameters obtained fitting the data of line $A$, blue. The red stars represent the data collected in laboratory, Table 1. From top to bottom: $P, F$. 
Table 2: The data set for line $B$

\begin{tabular}{c|c|c|c|c|c|c} 
Time $(\mathrm{h})$ & $t_{0}$ & 72 & 98.5 & 116 & 188 & 260 \\
\hline$F(\mathrm{~g})$ & $3 * 10^{-3}$ & $1.9 * 10^{-2}$ & $1.9 * 10^{-2}$ & $3 * 10^{-2}$ & $2.6 * 10^{-2}$ & $2.1 * 10^{-2}$ \\
$C(\mathrm{~g} / \mathrm{l})$ & 2.316 & 1.336 & 0.833 & 0.348 & 0.132 & 0.131
\end{tabular}

The data of line B are reported in Table 2 .

The model in the absence of $P$ simplifies to

$$
\frac{d F}{d t}=-f k_{C} \frac{C F}{h_{C}+C}, \quad \frac{d C}{d t}=-k_{C} \frac{C F}{h_{C}+C},
$$

which has the equilibrium $(F(\infty), 0)$ and furthermore $f C+F=f C(0)+F(0)=$ $f C(\infty)+F(\infty)=0+F(\infty)$, again giving the value of $F(\infty)$. Thus the conversion factor is

$$
f=\frac{F(\infty)-F(0)}{C(0)}=0.0077 .
$$

The fitting of the dataset from line B allows then the determination of the parameters

$$
k_{C}=2.9416(g s)^{-1}, \quad h_{C}=0.6296 \mathrm{~g} / \mathrm{l} .
$$

In Figure $2 F$ and $C$ are shown respectively for $260 \mathrm{~h}$ (10 days), with the red stars representing the real data collected in laboratory, while the blue continuous lines indicate the optimal solutions of the system, (7).
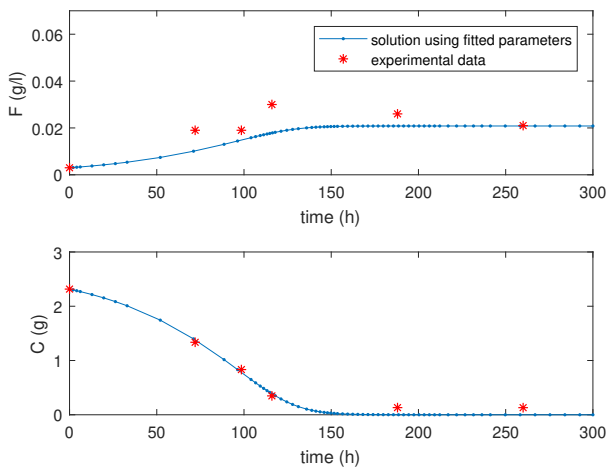

Figure 2: The solution of (1) using the parameters obtained fitting the data of line $B$, blue. The red stars represent the data collected in laboratory, Table 2. From top to bottom: $F, C$.

To fit the remaining two parameters, $a_{P}$ and $a_{C}$ we used the data of line C. As previously mentioned the initial conditions for $P$ and $F$ were comparable with line A $(0.24 \mathrm{~g} / 1$ and $0.003 \mathrm{~g}$, respectively) but the initial concentration of $C$ was higher $(2.35 \mathrm{~g} / \mathrm{l})$. In Table 3 we list the set of data obtained from 
the laboratory experiments for line C. The fungus grew 4-fold more than in line A. As regards $P$, the final degradation yields are comparable: both in line $\mathrm{A}$ and $\mathrm{C}$, at the end of the experiment the decolourisation of $P$ was higher than $90 \%$. However, the presence of $C$ in line $\mathrm{C}$ enhances the efficiency $(P$ transformation during t) of the system. The fungus was faster in the presence of glucose addition. In line $\mathrm{C}$ and $\mathrm{A}$, the color removal was respectively $35 \%$ and $15 \%$ after $16 \mathrm{~h}$, and, $70 \%$ and $25 \%$ after $48 \mathrm{~h}$.

Table 3: The data set for line $C$

\begin{tabular}{c|c|c|c|c|c|c|c|c|c} 
Time $(\mathrm{h})$ & $t_{0}$ & 1.5 & 16.5 & 19.5 & 22.5 & 27 & 41 & 45 & 48 \\
\hline$P(\mathrm{~g} / \mathrm{l})$ & 0.200 & 0.210 & 0.130 & 0.125 & 0.114 & 0.094 & 0.096 & 0.064 & 0.061 \\
$F(\mathrm{~g})$ & $3 * 10^{-3}$ & $4 * 10^{-3}$ & $5 * 10^{-3}$ & $6 * 10^{-3}$ & $7 * 10^{-3}$ & $3 * 10^{-3}$ & $12 * 10^{-3}$ & $9 * 10^{-3}$ & $9 * 10^{-3}$ \\
$C(\mathrm{~g} / \mathrm{l})$ & 2.35 & 2.35 & 2.30 & 2.46 & 2.26 & 2.28 & 2.07 & 2.14 & 1.95
\end{tabular}

\begin{tabular}{c|c|c|c|c|c|c|c|c} 
Time $(\mathrm{h})$ & 51 & 65 & 69 & 72 & 98.5 & 116 & 188 & 260 \\
\hline$P(\mathrm{~g} / \mathrm{l})$ & 0.053 & 0.038 & 0.031 & 0.024 & 0.023 & 0.023 & 0.026 & 0.019 \\
$F(\mathrm{~g})$ & $16 * 10^{-3}$ & $14 * 10^{-3}$ & $14 * 10^{-3}$ & $18 * 10^{-3}$ & $27 * 10^{-3}$ & $24 * 10^{-3}$ & $22 * 10^{-3}$ & $24 * 10^{-3}$ \\
$C(\mathrm{~g} / \mathrm{l})$ & 1.83 & 1.53 & 1.43 & 1.35 & 1.01 & 0.59 & 0.16 & 0.16
\end{tabular}

The values in Tables 1, 2 and 3 are the mean of three different measurements, the flasks are chosen randomly and then are sacrificed as described in the previous subsection. One can see that in line $\mathrm{C}$ the fungus grows until the 4 th day and then remains stable because $C$ is close to zero and $F$ cannot convert it into biomass anymore. The occasional very small drops in the fungus population are due in part to the limit of the detection of the precision analytical scale used, in part to the way data were collected during the experiment.

Furthermore we have chosen to measure our data more often at the beginning of the process to better capture the transient phase and then more rarely once the biodegradation reached the critical level at which no further decolourisation is possible. We can say that after 100 hours the process became "stable". Preliminary trials (data not shown) showed us that most of the decolourisation occurred in the first days of the experiment, but $C$ was not completely exhausted. In the present study, in order to describe more precisely the behavior of $F$ (in correlation to $C$ and $P$ ) we decided to continue the experiment for 7 days.

The optimization algorithm has been used to fit the laboratory data. It appears to be a bit sensitive to the initial guesses for the parameters. We have used a set of different initial randomly generated starting points for the parameters. The ones that gave the least value of the norm of the residual were selected as candidates and the final choice $(1,1)$ for the initial condition was made, because visually it provided the best fit, especially for the tail of the data points distribution. For line $\mathrm{C}$ we get the following possible set of parameters :

$$
a_{P}=2.4 * 10^{-14}(l s)^{-1}, \quad a_{C}=3.9072(l s)^{-1},
$$


where the other parameters of the model (1) were used, as given in (6), (5), (8) and (9), respectively. With these optimal values, integrating once more system (1), the solution is found, that shows good agreement with the laboratory experiments. In Figure $3 P, F$ and $C$ are shown respectively for $260 \mathrm{~h}$ (10 days), with the red stars representing the real data collected in the laboratory, while the blue continuous lines indicate the solutions of the system, (1).
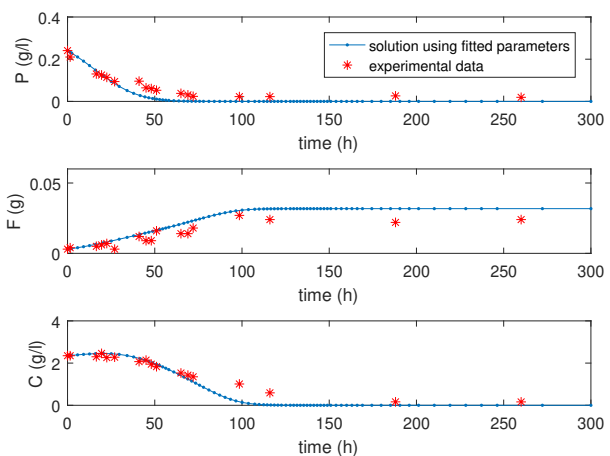

Figure 3: The solution of (1) using the parameters obtained fitting the data of line $C$, blue. The red stars represent the data collected in laboratory, Table 3. From top to bottom: $P, F, C$.

In Table 4 the set of best fit parameters for lines $A, B$ and $C$ are reported.

Table 4: Best fit parameters for the data obtained for line A, B and C

\begin{tabular}{c|c|c|c|c|c|c|c|c} 
Parameters & $a_{1}$ & $a_{P}$ & $h_{P}$ & $h_{C}$ & $e$ & $a_{C}$ & $k_{C}$ & \\
\hline lines $A, B, C$ & $664(l s)^{-1}$ & $2.4 * 10^{-14}(l s)^{-1}$ & $100 g / l$ & $0.6296 g / l$ & 0.022 & $3.9072(l s)^{-1}$ & $0.6296(g s)^{-1}$ & 0
\end{tabular}

Remark 1. The results of our experiments indicate that $a_{P}=2.4 * 10^{-14}$, see Table 4. This means that for the degradation process of the organic pollutants, the main role is obviously played by $\mathrm{F}$, whereas $\mathrm{C}$ is not directly acting on $\mathrm{P}$. Being a source of nutrients, $\mathrm{C}$ allows $\mathrm{F}$ to grow and be active, a fact that ultimately determines the transformation of P. Furthermore $a_{C}=3.9072>1$.

\section{The general mathematical model}

The system (1) introduced in Section 2 models a closed biodegradation system. In real life applications, reactors work in a continuous fashion, with a roundthe-clock feeding of waste water. In this section we therefore introduce a more general mathematical model in which we assume constant input rates of organic pollutant, $q_{P}$, and of glucose, $q_{C}$.

Since the time scale in this case is much larger than in the previous case, a new 
term representing the functional inactivity or mortality of the fungi population is introduced in the second equation. This occurs at a constant rate $m_{F}$. In [8] it was shown that once the process of degradation of the pollutants by the fungi starts, it is not necessary to have a constant input in time of fungi, thus we take here $q_{F}=0$. Recall that $a_{P}=2.4 * 10^{-14}$ and that $a_{C}>1$, in view of Remark 1, see Section 4 Table 4. For the general mathematical model we will assume $a_{P}=0$ and $a_{C}-1>0$. The general model reads

$$
\begin{aligned}
\frac{d P}{d t} & =q_{P}-a_{1} \frac{P}{h_{P}+P} F \\
\frac{d F}{d t} & =e a_{1} \frac{P}{h_{P}+P} F+f k_{C} \frac{C}{h_{C}+C} F-m_{F} F \\
\frac{d C}{d t} & =q_{C}-f k_{C} \frac{C}{h_{C}+C} F+\left(a_{C}-1\right) a_{1} \frac{P}{h_{P}+P} F .
\end{aligned}
$$

\section{The qualitative analysis of the general math- ematical model}

In this section the local stability analysis of the equilibrium points of the more general model (10) is performed. The equilibrium point is said to be locally asymptotically stable if properly choosing i.c. near it, the trajectories of the model will be attracted by the equilibrium. Furthermore, if specific conditions are needed to guarantee its stability, the equilibrium point is said to be conditionally locally asymptotically stable.

Solving the system obtained from model (10) we get just the coexistence equilibrium. Further, four additional equilibria exist if at least one among the constant supplies $q_{P}$ and $q_{C}$ is zero.

Proposition 2. If

$\left(a_{C}-1\right) a_{1} f q_{P}+a_{1} e q_{P}+a_{1} f q_{C}-m_{F} q_{P}>0$ and $\left[\left(a_{C}-1\right) q_{P}+q_{C}\right]\left(f k_{C}-m_{F}\right)+e k_{C} q_{P}>0$

the coexistence equilibrium

$E^{*}=\left(\frac{\left(a_{C}-1\right) f q_{P}+e q_{P}+f q_{C}}{m_{F}}, \frac{h_{P} m_{F} q_{P}}{\left(a_{C}-1\right) a_{1} f q_{P}+a_{1} e q_{P}+a_{1} f q_{C}-m_{F} q_{P}}, C^{*}\right)$.

with

$$
C^{*}=\frac{h_{C} m_{F}\left[\left(a_{C}-1\right) q_{P}+q_{C}\right]}{\left[\left(a_{C}-1\right) q_{P}+q_{C}\right]\left(f k_{C}-m_{F}\right)+e k_{C} q_{P}}
$$

is feasible; if the Routh-Hurwitz criteria hold

$$
A_{2}>0, \quad A_{3}>0 \text { and } A_{2} A_{3}>A_{4}
$$

where $A_{1}, A_{2}$ and $A_{3}$ are the coefficients of the cubic characteristic equation, $E^{*}$ is conditionally asymptotically stable.

Proof: Part 1: existence

The main steps to calculate the analytical expressions for $P^{*}, F^{*}$ and $C^{*}$ are: 
Step 1: We get $F$ from the first equilibrium equation of the system (10)

$$
F=\frac{q_{P}\left(h_{P}+P\right)}{a_{1} P}
$$

after substituting it into the second equilibrium equation of (10) we get

$$
\left[\left(\left(\left(a_{1} e+f k_{C}-m_{F}\right) P+h_{P}\left(f k_{C}-m_{F}\right)\right) C+h_{C}\left(\left(a_{1} e-m_{F}\right) P-h_{P} m_{F}\right)\right]=0 .\right.
$$

Step 2: Solving for $P$ equation (15) we have

$$
P=\frac{h_{P}\left(-C f k_{C}+C m_{F}+h_{C} m_{F}\right)}{\left(C a_{1} e+C f k_{C}+a_{1} e h_{C}-C m_{F}-h_{C} m_{F}\right)} .
$$

Step 3: Substituting $F$, from (14), and $P$, from (16), into the third equilibrium equation of (10) we get

$$
C^{*}=\frac{h_{C} m_{F}\left[\left(a_{C}-1\right) q_{P}+q_{C}\right]}{\left[\left(a_{C}-1\right) q_{P}+q_{C}\right]\left(f k_{C}-m_{F}\right)+e k_{C} q_{P}}
$$

that is always nonnegative if $\left[\left(a_{C}-1\right) q_{P}+q_{C}\right]\left(f k_{C}-m_{F}\right)+e k_{C} q_{P}>0$.

We thus find the coexistence equilibrium (12). For the nonnegativity of $P$ $\left(a_{C}-1\right) a_{1} f q_{P}+a_{1} e q_{P}+a_{1} f q_{C}-m_{F} q_{P}>0$ must be satisfied.

Part 2: stability

Further, for the stability analysis, we calculate the Jacobian matrix of the system (10):

$$
J=\left[\begin{array}{ccc}
-\frac{a_{1} h_{P} F}{\left(h_{P}+P\right)^{2}} & -\frac{a_{1} P}{h_{P}+P} & 0 \\
e \frac{a_{1} h_{P} F}{\left(h_{P}+P\right)^{2}} & e \frac{a_{1} P}{h_{P}+P}+\frac{f k_{C} C}{h_{C}+C}-m_{F} & +\frac{f k_{C} h_{C} F}{\left(h_{C}+C\right)^{2}} \\
\left(a_{C}-1\right) \frac{a_{1} h_{P} F}{\left(h_{P}+P\right)^{2}} & -\frac{k_{C} C}{h_{C}+C}+\left(a_{C}-1\right) \frac{a_{1} P}{h_{P}+P} & -\frac{k_{C} h_{C} F}{\left(h_{C}+C\right)^{2}}
\end{array}\right] .
$$

For $E^{*}$ we get a third degree polynomial

$$
\lambda^{3}+A_{2} \lambda^{2}+A_{3} \lambda+A_{4}=0
$$

with $A_{2}=\operatorname{tr} J\left(E^{*}\right), A_{3}$ is the sum of the principal minors of $J\left(E^{*}\right)$ and $A_{4}=$ $\operatorname{det} J\left(E^{*}\right)$. Their analytical expressions are too involved to be reported here. $E^{*}$ is stable if the Routh-Hurwitz conditions (13) hold. In Figure 4 we show that the coexistence equilibrium $E^{*}=(0.013,10,0.062)$ is stably achieved for the same set of parameters as for Figure 3, with $q_{P}=0.9, m_{F}=0.004$ and $q_{C}=0.02$.

\subsection{Sensitivity analysis}

Table 4 in Section 4 contains the values of the parameters for model (1) that can be determined from the experiments. In this section we perform a sensitivity 

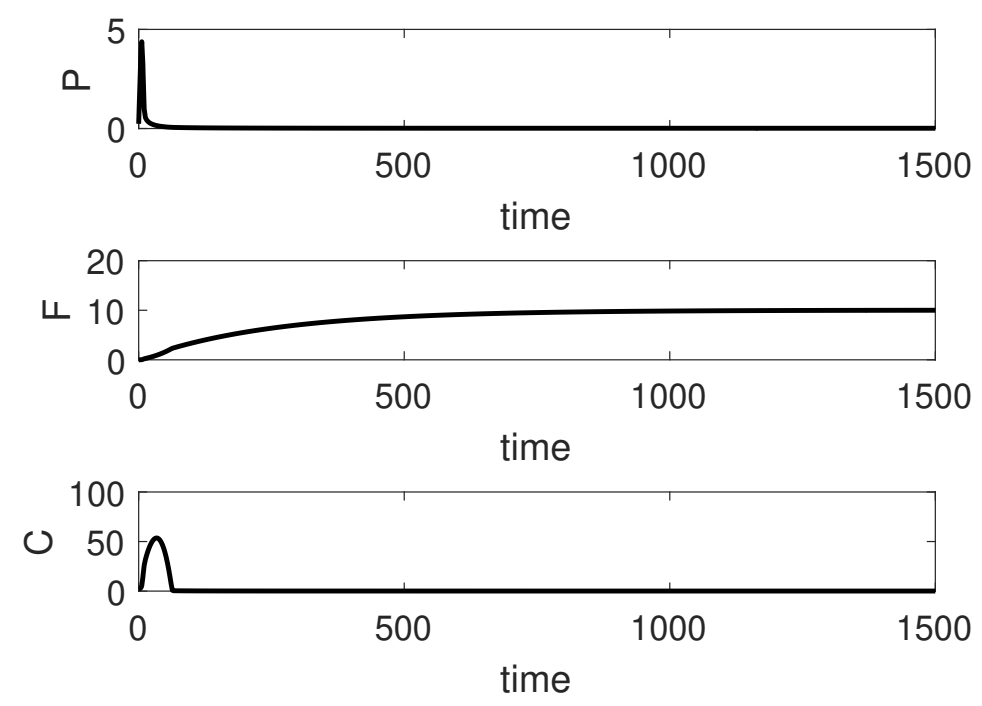

Figure 4: The stable coexistence equilibrium $E^{*}=(0.013,10,0.062)$ of $(10)$ is attained for the set of parameters obtained with the data of the line $B$. From top to bottom: $P, F, C$.

analysis for the still unknown parameters $q_{P}$ and $q_{C}$, namely the constant input of pollutants and of nutrients, respectively, and $m_{F}$, the inactivity rate of the fungi population. We will analyze the behaviour of the three populations of model (10) varying these parameters in pairs, $\left(q_{P}, q_{C}\right),\left(q_{P}, m_{F}\right)$ and $\left(q_{C}, m_{F}\right)$ respectively.

Figure 5 contains the changes of the equilibrium values of $P, F$ and $C$ if $q_{P}$ and $q_{C}$ assume different values in the interval $[0,10]$. In panel $(a)$ increasing the constant input of nutrients, $q_{C}$, the pollutant population decreases slightly while increasing the constant input of pollutant, $q_{P}$, the pollutant population, quite intuitively, increases. In panel $(b)$ the increase of $q_{C}$ and of the constant input of pollutant, $q_{P}$, leads to an increase in the fungi population. For the same value of $q_{P}$, there are two surfaces for $F$ and $P$, indicating the maximum and minimum values that these quantities attain. When they coincide, it means that a stable equilibrium is attained, while when they differ, the solution oscillates. In this case thus sustained oscillations in time are found. In the $(c)$ panel, increasing $q_{P}$ leads to the extinction of the nutrients population, $C$, meaning that they are used up in this degradation process. The parameter $q_{C}$ initially favors higher $C$ values, for low $q_{P}$ values, but larger parameter values keep them constant or even depress them slightly.

In Figure 6 we vary $\left(q_{P}, m_{F}\right)$ in the interval $[0,10] \times[0,1]$. In panel $(a)$, increasing the mortality (or inactivity) of the fungi population $m_{F}$ and $q_{P}$ leads to an increase of $P$, as we expect. Here note the axes reversal, for better illustration of the figure. Increasing $m_{F}$ leads to the extinction of $F$, panel $(b)$, while after a sudden initial surge, it has no effect on $C$. The parameter $q_{P}$ helps 


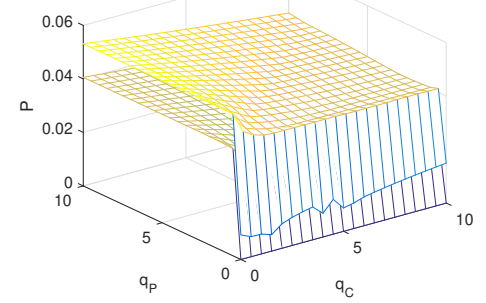

(a) Pollutant population varying $q_{P}$ and $q_{C}$.
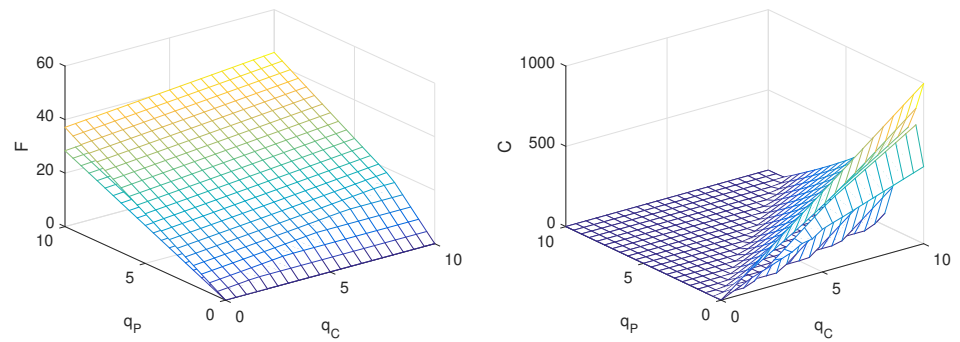

(b) Fungi population varying $q_{P}$ and(c) Nutrients population varying $q_{P}$ $q_{C}$. and $q_{C}$.

Figure 5: Steady state equilibria of $P, F$ and $C$ as functions of the model parameters $q_{P}$ and $q_{C}$ in the interval $[0,10]$. The i.c. $[0.24 ; 0.003 ; 2.35]$ and the parameters values as in Table 4 . When two different surfaces are shown they denote the maximum and minimum values of possible population oscillations.

in raising the fungi population, and of $C$. Once more, for the same value of $q_{P}$, there are two surfaces for $C$, panel $(c)$, indicating persistent oscillations.

Figure 7 shows the populations as functions of the parameters $\left(q_{C}, m_{F}\right)$ in $[0,10] \times[0,1]$. Increasing $q_{C}$ has no effect on $P$, panel $(a)$, and on $F$, panel $(b)$, but it leads to an increase in $C$, panel $(c)$. Increasing the parameter $m_{F}$ leads to an initial fast raise of $P$, to the vanishing of $F$ and does not apparently affect $C$. Again we can observe persistent oscillations for $P$ and $C$.

\section{Conclusions}

In this paper two mathematical models for the degradation of organic pollutants were introduced. One of them models a closed laboratory system where neither input nor output are present. The other one considers an open system, more resemblant to actual industrial water remediation reactors, with constant input of pollutant and glucose, respectively.

The model for the closed system has been used to fit the real data obtained 


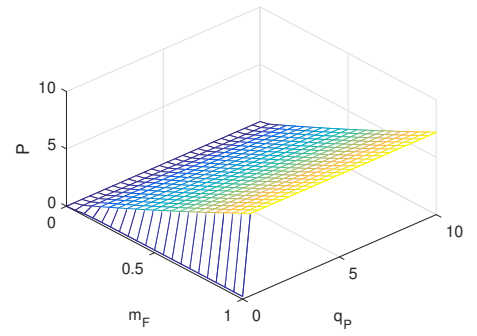

(a) Pollutant population varying $q_{P}$ and $m_{F}$.
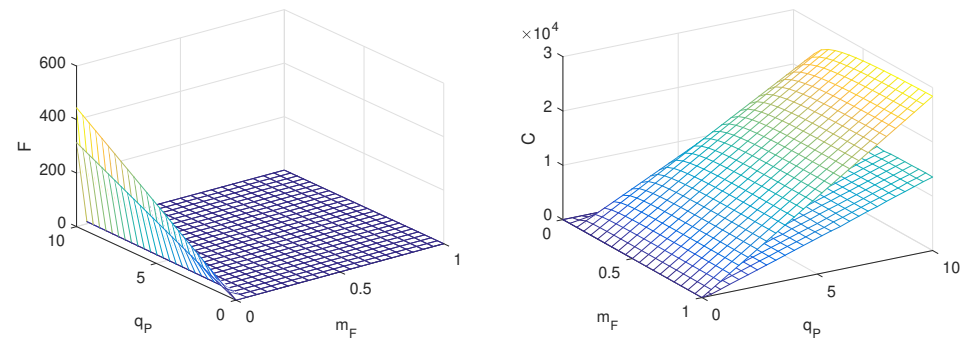

(b) Fungi population varying $q_{P}$ and(c) Nutrients population varying $q_{P}$ $m_{F}$. and $m_{F}$

Figure 6: $P, F$ and $C$ with $q_{P}$ and $m_{F}$ assuming different values in the interval $[0,10] \times[0,1]$. The i.c. $[0.24 ; 0.003 ; 2.35]$ and the parameters values as in Table 4. Note that in panel $a$ and $c$ the axes are rotated to better see the persistent oscillations.

in the laboratory. A set of parameters was found using the set of data obtained from the collected results, namely the experimental lines $A, B$ and $C$. Pollutants, fungi and glucose in the model are seen to behave in the same way as the real data. Trametes pubescens MUT 2400 confirms to be a good candidate to set biological treatments of colored wastewaters: it is capable of almost completely removing the colour. In the optimal conditions, most of the reaction already takes place within the first 2 days. It is therefore suitable to be involved in wastewater treatment plants that have to handle high volume of water in a restrained time. The system efficiency shows to be higher in the presence of low glucose addition (line $C$ ), reaching the same decolourisation yields in half time. These findings highlight the importance of having an even small amount of nutrients in the wastewaters in order to establish an effective and fast fungal treatment. Trametes pubescens is also seen to be a robust organism: it responds to almost starved cultural conditions (line $A$ ), by producing enzymes able to act against the pollutant. It could however be assumed that the activity of the fungus in this condition will decrease during time in an open system.

For the general mathematical model a coexistence equilibrium has been found 


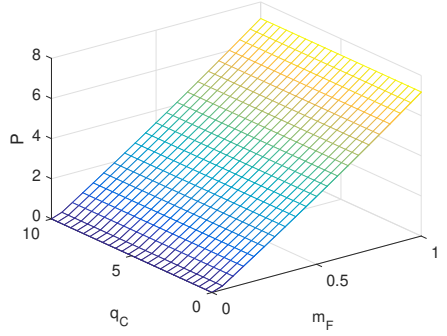

(a) Pollutant population varying $q_{C}$ and $m_{F}$.
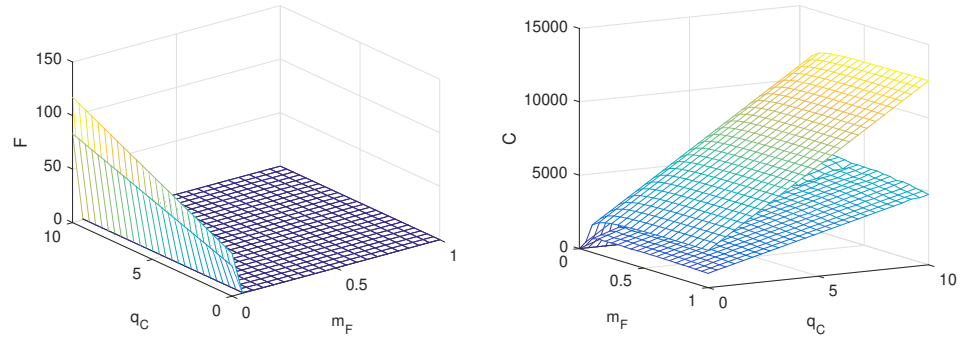

(b) Fungi population varying $q_{C}$ and(c) Nutrients population varying $q_{C}$ $m_{F}$. and $m_{F}$

Figure 7: $P, F$ and $C$ with $q_{C}$ and $m_{F}$ assuming different values in the interval $[0,10] \times[0,1]$. The i.c. $[0.24 ; 0.003 ; 2.35]$ and the parameters values as in Table 4 . Note that in panel $c$ the axes are rotated to better see the persistent oscillations.

and the conditions for its stability have been investigated. Using the set of parameters obtained from the data, Table 4, we have performed a simulation on the open system with continuous feeding to assess the behaviour of $P, F$ and $C$. The results reflect those obtained in the closed system, with the difference that although $C$ has a very low value, it does not completely disappear. These results are shown in Figure 4.

The sensitivity analysis of the model (10) with respect to $q_{P}$, the constant input of pollutant, $q_{C}$, the constant input of nutrients and $m_{F}$, the inactivity rate of fungi population shows that increasing $q_{P}$ while keeping $m_{F}$ at a constant value leads to an increase of the pollutant and nutrients populations respectively. For increasing inactivity rate of fungi population the latter get extinguished with only an indirect consequence on $P$ and $C$ respectively. Increasing $q_{C}$ leads to an increase of nutrients population if $m_{F}$ is constant. Increasing $m_{F}$ has no direct effect on $P$ and $C$ but it has an indirect one: since the fungi population goes extinct, the pollutant and the nutrients will increase by increasing the constant input of $P$ or the constant input of $C$.

It is our hope and expectation that the results of this investigation may help 
and support further larger scale experiments and finally the setting up of such digestors at an industrial scale.

Acknowledgment: The research has been partially supported by the project "Metodi numerici nelle scienze applicate" of the Dipartimento di Matematica "Giuseppe Peano".

IMB has been partially supported by "Finanziamento GNCS Giovani Ricercatori $2016 "$ ".

The authors wish to thank the anonymous reviewers for their valuable comments and suggestions to improve the quality of the paper.

\section{References}

[1] R. Kant, Textile dyeing industry an environmental hazard. R Natural Science 4 (2012) 22-26.

[2] M. Imran, D.E. Crowley, A. Khalid, S. Hussain, M.W. Mumtaz, M. Arshad, Microbial biotechnology for decolorization of textile wastewaters. Reviews in Environmental Science and Bio/Technology 14 (2016) 73-92 .

[3] K. Singh, S. Arora, Removal of synthetic textile dyes from wastewaters: a critical review on present treatment technologies. Critical Reviews in Environmental science and Technology 41 (2011) 807-878 .

[4] A. Anastasi, F. Spina, A. Romagnolo, V. Tigini, V. Prigione, G.C. Varese, Integrated fungal biomass and activated sludge treatment for textile wastewaters bioremediation. Bioresource Technology 123 (2012) 106-111 .

[5] I. Eichlerova, L. Homolka, F. Nerud, Decolorization of high concentration of synthetic dyes by the white rot fungus Bjerkandera adusta strain CCBAS 232. Dyes and Pigment 75 (2007) 38-44.

[6] P. Kaushik, A. Malik, Fungal dye decolorization: recent advances and future potential. Environmental International 35 (2009) 127-141 .

[7] I.M. Bulai, F. Spina, G. C. Varese, E. Venturino, Wastewater Bioremediation using White Rot Fungi: Validation of a Dynamical System. Biomath Communications, (2016).

[8] I.M. Bulai, E. Venturino, Biodegradation of organic pollutants in a water body. Journal of Mathematical Chemistry, (2016) 1387-1403.

[9] A. Goyal, R. Sanghi, A.K. Misra, J.B. Shukla, Modeling and analysis of the removal of an organic pollutant from a water body using fungi. Appl. Math. Model. 38 (2014) 4863-4871.

[10] A.K. Misra, Modeling the depletion of dissolved oxygen in a lake due to submerged macrophytes. Nonlinear Analysis: Modelling and Control 15(2) (2010) 185-198. 
[11] G.L. Miller, Use of dinitrosalicylic acid reagent for determination of reducing sugar. Analytical Chemistry 31 (1959) 426-428.

[12] F. Spina, C. Junghanns, I. Donelli, R. Nair, P. Demarche, A. Romagnolo, G. Freddi, S. N. Agathos and G. C. Varese, Stimulation of laccases from Trametes pubescens: Use in dye decolorization and cotton bleaching. Preparative Biochemistry and Biotechnology, 46:7 (2016) 639-647, DOI: 10.1080/10826068.2015.1128445.

[13] I.M.Bulai, E.Venturino, The Beddington-DeAngelis and the HTII product response functions: application to polluted ecosystems biodegradation. AIP Conf. Proc. 1738 (2016) 390002.

[14] I.M.Bulai, E.Venturino, Two mathematical models for dissolved oxygen in a lakeCMMSE-16. Journal of Mathematical Chemistry, 55(7) (2017) 14811504.

[15] C.P.Leslie Grady, Jr., Glen T. Daigeer, Nancy G. Love, Carlos D.M. Filipe, Biological wastewater treatment. Taylor and Francis Inc, (2011)

[16] R.D. McGee, J.F. Drake, A.G. Fredrickson, H.M. Tsuchiya, Studies in intermicrobial symbiosis, Saccharomyces cerevisiae and Lactobacillus casei. Can. J. Microbiol, 18 (1972) 1733-1742.

[17] S. Parekh, V.A. Vinci, R.J. Strobel, Improvement of microbial strains and fermentation processes. Appl Microbiol Biotechnol. 2000 Sep;54(3): 287-301.

[18] S. K. Sen, S. Raut, P. Bandyopadhyay, S. Raut, Fungal decolouration and degradation of azo dyes: A review, Fungal Biology Reviews, 30 (2016), 112133.

[19] S. Senthilkumar, M. Perumalsamy, H. Janardhana Prabhu, Decolourization potential of white-rot fungus Phanerochaete chrysosporium on synthetic dye bath effluent containing Amido black 10B, Journal of Saudi Chemical Society (2014) 18, 845-853. 